\title{
Classical Travelling Salesman Problem (TSP) based Approach to Solve Fuzzy TSP using Yager's Ranking
}

\author{
S.Dhanasekar \\ Research Scholar, Bharathiar \\ University, Asst. Prof. VIT \\ University, Chennai Campus
}

\author{
S.Hariharan,Ph.D \\ Assistant Professor \\ VIT University Chennai
}

\author{
P.Sekar,Ph.D \\ Principal \\ CKN College \\ Anna nager,Chennai
}

\begin{abstract}
Travelling salesman problem (TSP) is a classical and most widely studied problem in both operation research and computer science. If in the TSP the cost or time or distance is not certain then it is said to be Fuzzy TSP. We propose a new algorithm to solve the fuzzy TSP and also implemented the same and the results are discussed.
\end{abstract}

\section{General Terms}

Fuzzy TSP, Hungarian Method, Travelling Salesman Problem.

\section{Keywords}

Fuzzynumbers, fuzzy ranking, fuzzy assignment problem, fuzzy Hungarian method, route conditions.

\section{INTRODUCTION}

Travelling salesman problem is a classical problem in combinatorial optimization. Since 1950's it has been studied intensively. As a result of it, a large number of techniques were developed to solve the problem. The objective of the problem is to find the shortest route of salesman starting from a given city, visiting all other cities only once and finally come to the same city where he started. There are different approaches for solving travelling salesman problems. Linear programming method, heuristic methods like cutting plan algorithms and branch and bound method, markov chain, simulated annealing and tabu search methods. Few more algorithms like particle swarm optimization, neural networks, evolutionary computations, ant system, artificial bee colony, etc., are also there.

In real life situation it may not be possible to get the cost or time as certain quantity. To overcome this Zadeh [1] introduce fuzzy set concepts to deal with imprecision and vagueness. Since then significant advantages have been made in developing numerous methodologies and their applications to various decision problems. If the cost or time or distance is not crisp values, then it becomes a fuzzy TSP.

In recent years, Fuzzy TSP has got great attention and the problems in Fuzzy TSP have been approached using several technique .Hannan et al[2] solved Linear Programming Problem with multiple fuzzy goals. Hansen[3] applied tabu search algorithm. Jaszkiewicz [4] solved the problem by genetic local search algorithm. The evolutionary algorithm and dynamic search algorithm are discussed in Yan et al[5]., and Angel et al[6]., respectively. In Paraquete et al. [7], the extended local search algorithm called pareto local search algorithm was introduced. Liang et al [8] applied fuzzy multi objective linear programming for distribution planning decisions. In the paper of Rehemat et al.[9] the fuzzy linear programming was used to solve the problem. Jayadia. B et al [10] used fuzzy multi objective LP for No-wait flow shop scheduling. Tavakoli-Moghaddan.R et al [11] used fuzzy multi objective Linear programming to solve multi objective single machine scheduling problem. In the work of Mukerjee and Basu[12], a new method was proposed to solve fuzzy TSP. In the paper of Chaudri et al[13]., the fuzzy linear programming was used to solve the problem. Majumdar.J et al[14] used genetic algorithm to solve asymmetric TSP with fuzzy costs. Sepideh Fereidouni [15] approached the problem using multi objective linear programming. The Fuzzy TSP has been solved for LR-fuzzy parameters by Amit kumar and Anil gupta[16]. In this paper, a new algorithm which is similar to classical assignment method is introduced to solve fuzzy TSP. Firstly, Fuzzy Hungarian method is applied and then modifications are done to satisfy the route conditions by considering element wise subtraction and Yager's [17] ranking method of fuzzy numbers.

\section{PRELIMINARIES}

\subsection{Definition:}

The fuzzy set can be mathematically constructed by assigning a value to each possible individual in the universe of discourse to represent its grade of membership in the fuzzy set $[1,18]$.

\subsection{Definition:}

The fuzzy number $\widetilde{A}$ is a fuzzy set whose membership function $\mu_{\widetilde{\mathrm{A}}}(\mathrm{x})$ satisfies the following condition [7]
a) $\quad \mu_{\widetilde{\mathrm{A}}}(\mathrm{x})$ is a piecewise continuous
b) $\quad \mu_{\widetilde{A}}(x)$ is a convex
c) $?_{\widetilde{\mathrm{A}}}(\mathrm{x})$ is normal (i.e.) $\widetilde{\Omega}_{\widetilde{\mathrm{A}}}\left(\mathrm{x}_{0}\right)=1$.

\subsection{Definition:}

A fuzzy number with membership function in the form

$$
\mu_{\widetilde{A}}(x)=\left\{\begin{array}{l}
\frac{x-a}{b-a} \quad a \leq x \leq b \\
1 \quad x=b \\
\frac{c-x}{c-b} \quad b \leq x \leq c
\end{array}\right.
$$

is called a triangular fuzzy number $\widetilde{A}=(a, b, c)$ 


\subsection{Definition:}

A fuzzy number with membership function of the form

$$
\mu_{\widetilde{A}}(x)=\left\{\begin{array}{cc}
\frac{x-a}{b-a} & a \leq x \leq b \\
1 \text { b } & b x \leq c \\
\frac{d-x}{d-c} & c \leq x \leq d \\
0 \text { otherwise }
\end{array}\right.
$$

is called a Trapezoidal fuzzy number $\widetilde{A}=(a, b, c, d)$.

\subsection{Operations on trapezoidal and triangular fuzzy numbers:}

Addition:

$$
\begin{aligned}
\left(\mathrm{a}_{1}, \mathrm{~b}_{1}, \mathrm{c}_{1}, \mathrm{~d}_{1}\right)+\left(\mathrm{a}_{2}, \mathrm{~b}_{2}, \mathrm{c}_{2}, \mathrm{~d}_{2}\right) & =\left(\mathrm{a}_{1}+\mathrm{a}_{2}, \mathrm{~b}_{1}+\mathrm{b}_{2}, \mathrm{c}_{1}+\mathrm{c}_{2}, \mathrm{~d}_{1}+\mathrm{d}_{2}\right) \\
\left(\mathrm{a}_{1}, \mathrm{~b}_{1}, \mathrm{c}_{1}\right)+\left(\mathrm{a}_{2}, \mathrm{~b}_{2}, \mathrm{c}_{2},\right) & =\left(\mathrm{a}_{1}+\mathrm{a}_{2}, \mathrm{~b}_{1}+\mathrm{b}_{2}, \mathrm{c}_{1}+\mathrm{c}_{2}\right)
\end{aligned}
$$

Subtraction:

$$
\begin{gathered}
\left(a_{1}, b_{1}, c_{1}, d_{1}\right)-\left(a_{2}, b_{2}, c_{2}, d_{2}\right)=\left(a_{1}-d_{2}, b_{1}-c_{2}, c_{1}-b_{2}, d_{1}-a_{2}\right) \\
\left(a_{1}, b_{1}, c_{1}\right)-\left(a_{2}, b_{2}, c_{2},\right)=\left(a_{1}-c_{2}, b_{1}-b_{2}, c_{1}-a_{2}\right)
\end{gathered}
$$

\subsection{Element wise subtraction:}

$\left(a_{1}, a_{2}, a_{3}, a_{4}\right)-\left(b_{1}, b_{2}, b_{3}, b_{4}\right)=\left(a_{1}-b_{1}, a_{2}-b_{2}, a_{3}-b_{3}, a_{4}-b_{4}\right)$

$\left(a_{1}, a_{2}, a_{3}\right)-\left(b_{1}, b_{2}, b_{3}\right)=\left(a_{1}-b_{1}, a_{2}-b_{2}, a_{3}-b_{3}\right)$

\subsection{Yager's ranking method:}

The Yager's ranking is defined as

$\mathbf{Y}(\tilde{\mathbf{a}})=\int_{\mathbf{0}}^{\mathbf{1}}(\mathbf{0 . 5})\left(\mathbf{a}_{\boldsymbol{\alpha}}^{\mathrm{L}}+\mathbf{a}_{\boldsymbol{\alpha}}^{\mathrm{U}}\right) \mathbf{d} \boldsymbol{\alpha}$, where $\mathrm{a}_{\alpha}^{\mathrm{L}}=$ Lower $\alpha$ - level cut, $\mathrm{a}_{\alpha}{ }^{\mathrm{U}}=$ Upper $\alpha$ - level cut.

Yager's ranking technique satisfies compensation, linearity and additive property which provides results that are consistent with human intuition. If $Y(\tilde{\mathbf{s}}) \leq \mathrm{Y}(\tilde{\mathrm{I}})$ then $\tilde{\mathbf{s}} \leq \tilde{\mathrm{i}}$.

\subsection{Theorem:}

The crisps value of subtraction of two fuzzy numbers and crisps values of element wise subtraction of two fuzzy numbers are equal

Proof: Consider the fuzzy numbers $\left(\mathrm{a}_{1}, \mathrm{a}_{2}, \mathrm{a}_{3}, \mathrm{a}_{4}\right)$ and $\left(\mathrm{b}_{1}, \mathrm{~b}_{2}, \mathrm{~b}_{3}, \mathrm{~b}_{4}\right)$. Their subtraction is given by

$\left(a_{1}, a_{2}, a_{3}, a_{4}\right)-\left(b_{1}, b_{2}, b_{3}, b_{4}\right)=\left(a_{1}-b_{4}, a_{2}-b_{3}, a_{3}-b_{2}, a_{4}-b_{1}\right)$.

Crisp value of the fuzzy number is

$\mathrm{Y}\left(\mathrm{a}_{1}-\mathrm{b}_{4}, \mathrm{a}_{2}-\mathrm{b}_{3}, \mathrm{a}_{3}-\mathrm{b}_{2}, \mathrm{a}_{4}-\mathrm{b}_{1}\right)=\left(\mathrm{a}_{1}+\mathrm{a}_{2}+\mathrm{a}_{3}+\mathrm{a}_{4}\right)-$

$\left(b_{1}+b_{2}+b_{3}+b_{4}\right) / 4$ by using $(2.7)$.

The crisp value of element wise subtraction is

$\mathrm{Y}\left(\mathrm{a}_{1}-\mathrm{b}_{1}, \mathrm{a}_{2}-\mathrm{b}_{2}, \mathrm{a}_{3}-\mathrm{b}_{3}, \mathrm{a}_{4}-\mathrm{b}_{4}\right)=\left(\mathrm{a}_{1}+\mathrm{a}_{2}+\mathrm{a}_{3}+\mathrm{a}_{4}\right)-\left(\mathrm{b}_{1}+\mathrm{b}_{2}+\mathrm{b}_{3}+\mathrm{b}_{4}\right) / 4$

The crisp values are same when we use fuzzy subtraction and element wise subtraction.

\subsection{Fuzzy Travelling Salesman problem:}

It can be stated in the form of $n \times n$ cost matrix city1 city2 . cityN

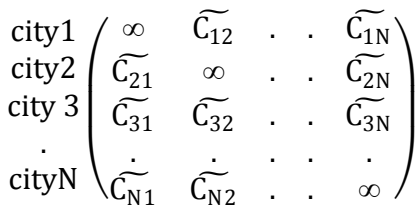

Mathematically it can be stated as Minimize $\tilde{Z}=\sum_{i=1}^{n} \sum_{j=1}^{n} \widetilde{\mathrm{C}_{i j}} x_{i j}$ subject to

$$
\begin{aligned}
& \sum_{\mathrm{i}=1}^{\mathrm{n}} \mathrm{x}_{\mathrm{ij}}=1, \mathrm{j}=1,2 \ldots \ldots \mathrm{n} \text {, } \\
& \sum_{j=1}^{n} x_{i j}=1, i=1,2 \ldots \ldots . n \\
& \mathrm{x}_{\mathrm{ij}}+\mathrm{x}_{\mathrm{ji}} \leq 1,1 \leq \mathrm{i} \neq \mathrm{j} \leq \mathrm{n} \\
& \mathrm{x}_{\mathrm{ij}}+\mathrm{x}_{\mathrm{jk}}+\mathrm{x}_{\mathrm{ki}} \leq 2,1 \leq \mathrm{i} \neq \mathrm{j} \neq \mathrm{k} \leq \mathrm{n} \\
& \mathrm{x}_{\mathrm{ip}_{1}}+\mathrm{x}_{\mathrm{p}_{1} \mathrm{p}_{2}}+\cdots+\mathrm{x}_{\mathrm{p}_{\mathrm{n}-2} \mathrm{i}} \leq \mathrm{n}-2 \text {, } \\
& \mathrm{p}_{1} \neq \mathrm{p}_{2} \neq \cdots \mathrm{p}_{\mathrm{n}-2} \leq \mathrm{n}
\end{aligned}
$$

Here (1) and (2) ensures that each city is visited only once. (3) Eliminates all 2-city sub tours and (4) eliminates all 3city sub tours. Finally, (5) eliminate all (n-2) city sub tours.

For feasibility solution of a TSP it should not contain sub tours.

\section{FUZZY HUNGARIAN METHOD \\ 3.1 Procedure}

Step: 1

Subtract the minimum of each row of the effectiveness matrix, from all the fuzzy elements of the respective rows. (Subtract means element wise subtraction).

Step: 2

Further modify the resulting matrix by subtracting the minimum element of each column from all the elements of the respective column. Thus obtained first modified matrix. (Subtract means element wise subtraction)

Step: 3

Then draw the minimum no of horizontal and vertical lines to cover all the fuzzy zero elements in the resulting matrix. If these may be two possibilities a) if $\mathrm{N}=\mathrm{n}$ (number of rows) then an optimal assignment can be made. So make the zero assignment to get the required solution. b) If $\mathrm{N}<\mathrm{n}$, then proceed to step 4

Step: 4

Determine the smallest fuzzy element in the matrix not covered by $\mathrm{N}$ lines. Use extension of addition method to all uncovered elements and add the same element at the intersection of horizontal and vertical lines. Thus the second modified matrix is obtained.

Step: 5

Again repeat the steps 3 and 4 until minimum no of lines become equal to the number of rows or columns (i.e.) $\mathrm{N}=\mathrm{n}$. 
Step: 6

Examine the rows successively until a row-wise exactly single fuzzy zero element is found. Mark this fuzzy zero element by ( ) to make the assignment, then crossover all fuzzy zero elements is lying in the respective column, showing that they cannot be considered for future assignment. Continue in this manner until all the rows have been examined.

Step: 7

Repeat the step 6 successively until one of the following situation arises:

a) if no unmarked fuzzy zero element is left, then the process ends or

b) if there lie more than one unmarked fuzzy zero elements in any column or row, then mark the one of the unmarked fuzzy zero elements arbitrary and a cross in the cells of remaining fuzzy zero elements in its row or column. Repeat the process until no unmarked fuzzy zero element is left in the matrix.

Step: 8

Thus exactly one marked fuzzy zero element in each row and each column of the fuzzy cost matrix is obtained

The assignment corresponding to these marked fuzzy zero elements gives the optimal assignment.

Step: 9

Scrutinizing the solution obtained under to see if the route conditions are satisfied. If it satisfies that's the solution of fuzzy TSP. If not, making adjustments in assignments to satisfy the condition with minimum increase in total cost (i.e.) to satisfy route condition "next best solution" may require considering.

\section{NUMERICAL EXAMPLES}

\subsection{Example:}

Consider the following Fuzzy Transportation Problem discussed in [17]:

$\left(\tilde{C}_{i j}\right)=\left(\begin{array}{cccc}\infty & \langle 91013\rangle & \langle 6835\rangle & \langle 8913\rangle \\ \langle 9,10,2,4\rangle & \infty & \langle 10,11,3,1\rangle & \langle 4,5,1,3\rangle \\ \langle 7813\rangle & \langle 101134\rangle & \infty & \langle 7823\rangle \\ \langle 91035\rangle & \langle 91134\rangle & \langle 6815\rangle & \infty\end{array}\right)$

Solution: After applying row reduction and column reduction using the element wise subtraction, we get the following matrix

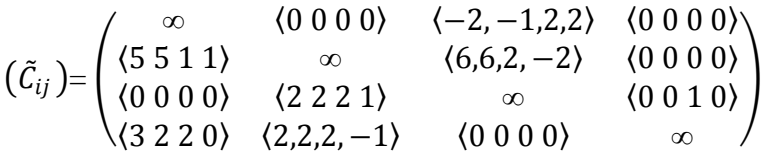

Here the minimum number of lines $=$ number of rows.

Therefore, the assignment is represented by

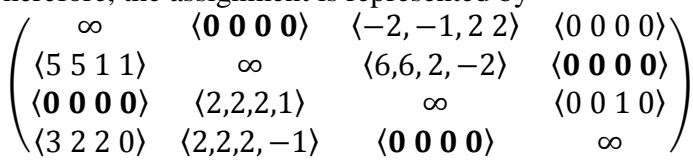

$\mathrm{A} \rightarrow \mathrm{B} \rightarrow \mathrm{D} \rightarrow \mathrm{C} \rightarrow \mathrm{A}$. Thus route conditions are satisfied. The fuzzy optimal total cost is

$\langle 91013\rangle+\left\langle 4 \begin{array}{llll}5 & 13\rangle+\langle 7813\rangle+\langle 68 & 1 & 5\end{array}\right\rangle=\langle 2631414\rangle$.

\subsection{Example}

Consider the following Fuzzy TSP discussed in [13] and [15]:

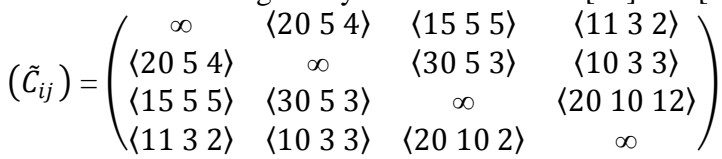

Solution: The resultant matrix after applying the row reduction and column reduction using the new kind of subtraction, we obtain

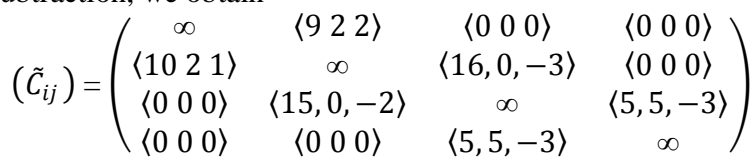

The minimum number of lines $=$ number of rows. Therefore, the assignment is represented by

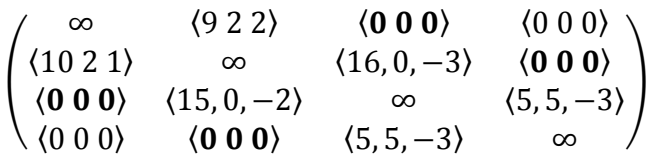

$\mathrm{A} \rightarrow \mathrm{C} \rightarrow \mathrm{A}, \mathrm{B} \rightarrow \mathrm{D} \rightarrow \mathrm{B}$. Thus route conditions are NOT satisfied. We have to go for next best solution.

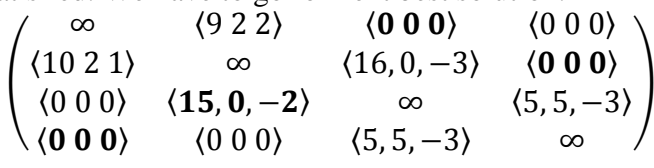

$\mathrm{A} \rightarrow \mathrm{C} \rightarrow \mathrm{B} \rightarrow \mathrm{D} \rightarrow \mathrm{A}$. The route conditions are satisfied The fuzzy optimal total cost is $=$

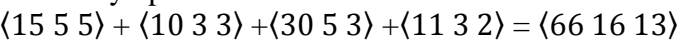
The next best solution is

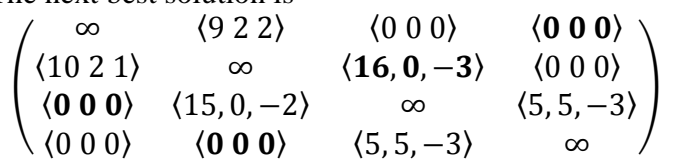

$\mathrm{A} \rightarrow \mathrm{D} \rightarrow \mathrm{B} \rightarrow \mathrm{C} \rightarrow \mathrm{A}$. The route condition are satisfied. The fuzzy optimal total cost is

$\langle 1555\rangle+\langle 1033\rangle+\langle 3053\rangle+\langle 1132\rangle=\langle 661613\rangle$.

Since both have same value both path are optimal path.

\section{CONCLUSION}

In this paper, a new algorithm for solving fuzzy TSP is introduced. This algorithm is effective and easy to understand because of its natural similarity to classical method of solving TSP. The examples shown in this paper guarantees the correctness and effectiveness of the working procedure of the algorithm. The results match with the existing technique and also satisfy the regular TSP conditions

\section{REFERENCES}

[1] Bellman RE, Zadeh LA .1970. Decision-making in a fuzzy environment, Manage. Sci., 17: 141-164.

[2] Hannan EL 1981. Linear programming with multiple fuzzy goals. Fuzzy Sets Syst., 6: 235-248

[3] Hansen MP 2000. Use of substitute Scalarizing Functions to guide Local Search based Heuristics: The case of MOTSP, J. Heuristics, 6: 419-431

[4] Jaszkiewicz A 2002. Genetic Local Search for Multiple Objectives Combinatorial Optimization. Eur. J. Oper. Res., 137(1): 50-71. 
[5] Yan Z, Zhang L, Kang L, Lin G 2003. A new MOEA for multi-objective TSP and its convergence property analysis. Proceedings of Second International Conference, Springer Verlag, Berlin, pp. 342-354.

[6] Angel E, Bampis E, Gourvès, L 2004. Approximating the Pareto curve with Local Search for Bi-Criteria TSP $(1,2)$ Problem, Theor. Comp.Sci., 310(1-3): 135-146.

[7] Paquete L, Chiarandini M, Stützle T 2004. Pareto Local Optimum Sets in Bi-Objective Traveling Sales man Problem: An Experimental Study. In: Gandibleux X., Sevaux M., Sörensen K. and Tkindt V. (Eds.), Metaheuristics for Multi-objective Optimization. Lect. Notes Ec on. Math. Syst., Springer Verlag, Berlin, 535: 177-199.

[8] Liang TF 2006. Distribution planning decisions using interactive fuzzy multi-objective linear programming. Fuzzy Sets Syst., 157: 1303-1316.

[9] Rehmat A, Saeed H, Cheema MS 2007. Fuzzy Multiobjective Linear Programming Approach for Traveling Salesman Problem. Pak. J. Stat. Oper. Res., 3(2): 87-98.

[10] Javadia B, Saidi-Mehrabad M, Haji A, Mahdavi I, J olai F, Mahdavi-Amiri .N 2008. No-wait flow shop scheduling using fuzzy multi-objective linear programming. J. Franklin Inst., 345: 452-467.

[11] Tavakoli-Moghaddam R, Javadi B, J olai F, Ghodratnama A 2010. The use of a fuzzy multi-objective linear programming for solving a multi-objective singlemachine scheduling problem. Appl. Soft Comput., 10: 919-925.

[12] Mukherjee S. and Basu, K. 2010. Application of fuzzy ranking method for solving assignment problems with fuzzy costs. International Journal of Computational and Applied Mathematics, 5: 359-368.

[13] Chaudhuri A, De K 2011. Fuzzy multi-objective linear programming for traveling sales man problem. Afr. J. Math. Comp. Sci. Res., 4(2): 64-70.

[14] Majumdar J, Bhunia AK 2011. Genetic algorithm for asymmetric traveling salesman problem with imprecise travel times. J. Comp. Appl. Math., 235: 3063-3078.

[15] Sepideh Fereidouni 2011. Travelling salesman problem by using a fuzzy multi-objective linear programming. African Journal of mathematics and computer science research 4(11) 339-349

[16] Amit kumar and Anil gupta, 2012. Assignment and Travelling salesman problems with co-efficient as LR fuzzy parameter. International Journal of applied science and engineering 10(3) 155-170.

[17] R.R.Yager, 1981 .A procedure for ordering fuzzy subsets of the unit interval. Information Sciences, 24, 143-161

[18] Zadeh LA 1965. Fuzzy Logic and its Applications, Academic Press, New York 